\title{
SEPARATING THE SECULAR MOTION OF THE POLE FROM CONTINENTAL DRIFT \\ WHERE AND WHAT TO OBSERVE?
}

\author{
IVAN I. MUELLER and C. R. SCHWARZ \\ Department of Geodetic Science, The Ohio State University, Columbus
}

\begin{abstract}
The paper estimates the expected rates of changes in the coordinates of various observatory networks (IPMS, BIH, VLBI, etc.) due to the secular motion of the pole and/or continental drift according to the Le Pichon Model. Based on these estimates, suggestions are made as to what to observe and over what time span to separate the effect of continental drift and secular polar motion.
\end{abstract}

\section{Introduction}

Although palaeomagnetic and other evidence argue strongly for the view that the continents have drifted apart from each other during geologic times, there has not yet been any geodetic determination of whether continental drift is occurring at the present time. Since it seems likely that the drifting motions of the continents are continuous rather than catastrophic in nature, detection of the present rates of continental drift will be a major goal of geodesy in the future. The accuracies of the station positions presently obtained from geodetic techniques are of the order of a few meters. However, it is probable that future observations will be sufficiently precise to detect motions of the continents relative to the pole and/or to each other, especially if highly precise geodetic networks are established and then reobserved after a period of several years or decades.

The purpose of this study was to predict the magnitude and direction of the motion to be expected at various geodetic and astronomical observatories, so that a judgment might be made of the time base necessary to detect continental drift.

There are several kinds of observations to be considered. First, the Very Long Baseline Interferometry (VLBI), which will define a few highly precise baselines whose length and orientation will be determined with precisions as high as one part in one hundred million. The second group of observations to be considered is the optical and laser observations of geodetic satellites and/or of the Moon analyzed in the geometric (simultaneous) mode. These and the VLBI observations are ideal to determine the relative motions of the continents. For a complete description of these relative motions, the network should include at least three stations on each tectonic block. Optical and laser observations analyzed in the dynamic mode constitute a third group especially useful to detect motions in the absolute sense, i.e., relative to the Conventional International Origin (CIO). Similar purpose is served by the fourth group, the astronomic observations of observatories associated with the IPMS and the BIH which observe astronomic latitude and/or longitude. In order to separate continental drift from secular polar motion, both relative and absolute observations are needed. 


\section{Assumptions}

The basic assumption used in this study was the geometrical model of continental drift hypothesized by Le Pichon (1968). According to this model, the surface of the Earth consists of six large rigid plates in motion relative to each other. The only modifications of the rigid blocks occur along some or all of their boundaries. These boundaries are the crests of the mid-ocean ridges, and their associated transform faults, and the active trenches and regions of active folding or thrusting. The relative displacement of any block with respect to another is represented as a rotation on the spherical surface of the Earth. That is, the spreading zones all lie along arcs of great circles, while the direction of motion and the transform faults lie along small circles perpendicular to the spreading zones. The spreading occurs around some pole of rotation which is associated with the spreading zone. Le Pichon used five principal spreading zones (and associated poles) and showed that the observed spreading rates and azimuths of the transform faults fit this model reasonably well. These spreading zones were then assumed to have known rates of opening (Table I) and the rates of spreading or compression were then computed for the other boundaries of the six rigid blocks.

TABLE I

Centers of rotation for the five principal spreading zones (from Le Pichon, 1968)

\begin{tabular}{llcc} 
Pole & $\begin{array}{l}\text { Latitude } \\
(\mathrm{deg})\end{array}$ & $\begin{array}{l}\text { Longitude } \\
(\mathrm{deg})\end{array}$ & $\begin{array}{l}\text { Angular rate } \\
\left(10^{-7} \mathrm{deg} / \mathrm{yr}\right)\end{array}$ \\
\hline A. South Pacific (Antarctica-Pacific) & $70 \mathrm{~S}$ & $118 \mathrm{E}$ & 10.8 \\
B. Atlantic (America-Africa) & $58 \mathrm{~N}$ & $37 \mathrm{~W}$ & 3.7 \\
C. North Pacific (America-Pacific) & $53 \mathrm{~N}$ & $47 \mathrm{~W}$ & 6.0 \\
D. Indian Ocean (Africa-India) & $26 \mathrm{~N}$ & $21 \mathrm{E}$ & 4.0 \\
E. Arctic Ocean (America-Eurasia) & $78 \mathrm{~N}$ & $102 \mathrm{E}$ & 2.8
\end{tabular}

The Le Pichon model is expressed in terms of the relative movements of the six rigid blocks. However, it fails to predict the absolute motion of a point with respect to the solid body of the Earth or with respect to the CIO. In order to determine how each block moves with respect to a coordinate system fixed in the solid Earth, it is necessary to assume that one of the blocks remains fixed. For this purpose we chose the Antarctic block. There is no compelling reason for choosing the Antarctic block, since at present there seems to be no way of determining absolute motion. However, the fixing of Antarctica does fit the concept of Antarctica as the remnant of a protocontinent in the southern hemisphere from which South America, Africa, India, and Australia drifted northward. If the Antarctic block is not fixed with respect to the solid Earth, then, in the concept of this study, the periodic mean pole of rotation is carried away from the CIO, thus an apparent secular motion of the pole is evidenced. Conversely, any observed secular motion of the pole can be eliminated in the concept of assigning this motion to the Antarctic block. 
The latitude, longitude, and spreading rates associated with the centers of rotation given by Le Pichon may be considered as the polar coordinates of an angular velocity vector which characterizes the spreading motion. Thus, if $X$ is the position vector of a point on the North Atlantic rise and $\Omega_{\mathrm{E}}$ is the angular velocity vector associated with the Arctic Ocean center of rotation (the subscript refers to the ordering in Table I), then $\Omega_{\mathrm{E}} \times X$ is the linear velocity vector giving the relative velocity between the American and Eurasian blocks at that point. Similarly, the expression $\Omega_{\mathrm{A}} \times X$ will give the velocity, relative to Antarctica, of a point on the Pacific side of the South Pacific spreading zone. Since the Antarctic block is assumed to be fixed, this is also the absolute motion (relative to the solid Earth). Since this expression is valid for all points along the spreading zone, it must be valid for the whole Pacific block. Thus, $\Omega_{\mathrm{A}}$ is the angular velocity vector characterizing the rotational movement of the Pacific block. Since the angular velocities add vectorially, the angular velocities for the other blocks can also be worked out in this manner. The angular velocity vectors for the six rigid blocks, expressed in polar coordinates, are given in Table II.

TABLE II

Angular velocities of the six rigid blocks (relative to Antarctica)

\begin{tabular}{llllr}
\hline Block & Equation & $\begin{array}{l}\text { Latitude } \\
(\mathrm{deg})\end{array}$ & $\begin{array}{l}\text { Longitude } \\
(\mathrm{deg})\end{array}$ & $\begin{array}{l}\text { Angular rate } \\
\left(10^{-7} \mathrm{deg} / \mathrm{yr}\right)\end{array}$ \\
\hline 1. Antarctica & $\Omega_{1}=0$ & - & - & \\
2. Pacific & $\Omega_{2}=\Omega_{\mathrm{A}}$ & $70 \mathrm{~S}$ & $118 \mathrm{E}$ & 10.8 \\
3. America & $\Omega_{3}=\Omega_{\mathrm{A}}+\Omega_{\mathrm{C}}$ & $79.9 \mathrm{~S}$ & $40.4 \mathrm{E}$ & 5.4 \\
4. Eurasia & $\Omega_{4}=\Omega_{\mathrm{A}}+\Omega_{\mathrm{C}}+\Omega_{\mathrm{E}}$ & $62.9 \mathrm{~S}$ & $70.0 \mathrm{E}$ & 2.9 \\
5. Africa & $\Omega_{\mathrm{5}}=\Omega_{\mathrm{A}}+\Omega_{\mathrm{C}}+\Omega_{\mathrm{B}}$ & $43.2 \mathrm{~S}$ & $13.7 \mathrm{~W}$ & 3.2 \\
6. India & $\Omega_{6}=\Omega_{\mathrm{A}}+\Omega_{\mathrm{C}}+\Omega_{\mathrm{B}}+\Omega_{\mathrm{D}}$ & $4.6 \mathrm{~S}$ & $7.4 \mathrm{E}$ & 5.7
\end{tabular}

From these block motions, the relative motion along all block boundaries may be computed by taking the difference between the vectors associated with the respective blocks. By this method, Le Pichon computed his Table IV (Le Pichon, 1968, p. 3676, and Le Pichon, 1970).

\section{Predicted Motions}

With the model described in the previous section, it is possible to predict either the absolute motion of a single station or the relative motion of a pair of stations. Let station $i$ be located on block $k$ and let $\Omega_{k}$ be the components of the angular velocity vector associated with block $k$, resolved in Cartesian terrestrial coordinates. The components of the angular velocity vector, resolved in local coordinates at the station, can be obtained by a series of orthogonal matrix transformations (Arur and Mueller, 1971). Thus,

$$
R_{2}\left(\varphi_{i}\right) R_{3}\left(\lambda_{i}\right) \Omega_{k}=\left(\begin{array}{c}
-\dot{A}_{i} \\
-\dot{\varphi}_{i} \\
\cos \varphi_{i} \dot{\lambda}_{i}
\end{array}\right)
$$


where $\varphi_{i}$ and $\lambda_{i}$ denote the latitude and longitude at station $i$, and $\dot{A}_{i}$ denotes the variation in azimuth at the station. The rates of change of latitude and longitude, $\dot{\varphi}_{i}$ and $\dot{\lambda}_{i}$, may be taken directly from this equation.

The linear velocity at any station is given by $V=\Omega \times X$. Let $\Delta V$ be the relative velocity vector between two stations, and let $\Delta X$ be their relative position vector. Further, let $\alpha, \beta$, and $D$ be the polar coordinates of the point whose Cartesian coordinates are the components of $\Delta X$, i.e.,

$$
\begin{aligned}
& \alpha=\tan ^{-1}(\Delta y / \Delta x) \\
& \beta=\sin ^{-1}(\Delta z / D) \\
& D=|\Delta X|=\left(\Delta x^{2}+\Delta y^{2}+\Delta z^{2}\right)^{1 / 2} .
\end{aligned}
$$

Then $\alpha$ is the longitude direction component of the line between the two stations, $\beta$ is the latitude direction component, and $D$ is the length of the line. The rates of change of these components may be obtained from the equation

$$
R_{2}\left(90^{\circ}-\beta\right) R_{3}(\alpha) \Delta V=\left(\begin{array}{c}
-D \dot{\beta} \\
D \cos \beta \dot{\alpha} \\
\dot{D}
\end{array}\right)
$$

Thus $\dot{D}$ gives the predicted change in the length of a baseline between two stations on different blocks, and $\dot{\theta}=\left(\dot{\beta}^{2}+\cos ^{2} \beta \dot{\alpha}^{2}\right)^{\frac{1}{2}}$ gives the total rate of change of the orientation of the line.

It is also of interest to predict the expected changes in latitude and longitude at a station due to secular motion of the pole. Secular motion of the pole at a rate of $a_{p}$ and in a direction $\alpha_{p}$ may be viewed as an angular velocity of the crust with respect to the solid Earth (Arur and Mueller, 1971). In the terrestrial coordinate system, the components of the angular velocity vector of this motion are

$$
\Omega_{p}=\left(\begin{array}{c}
a_{p} \sin \alpha_{p} \\
-a_{p} \cos \alpha_{p} \\
0
\end{array}\right)
$$

As with the angular velocity due to continental drift, the components of this vector may be transformed into a local coordinate system at any station, and the expected rates of change in latitude and longitude, $\dot{\varphi}$ and $\dot{\lambda}$, may be computed. For the numerical predictions, the value of $\alpha_{p}$ was $285^{\circ}$ (Mueller, 1969, p. 82), and the value of $a_{p}$ was 0"0033 per year (Arur and Mueller, 1971).

The predicted motions at the observing stations for certain types of observations are shown in Figures 1, 2, and 3. Figure 1 shows the predicted rates of change of the length and orientation of selected VLBI baselines. Although the VLBI's promise to provide the most precise measurement of motion between continents, all stations participating at present in VLBI observations are located on only three of the six tectonic blocks, mostly in America and Europe (unfortunately, a similar statement is true for the lunar ranging stations). The line from Penticton to Parkes, Australia, has 


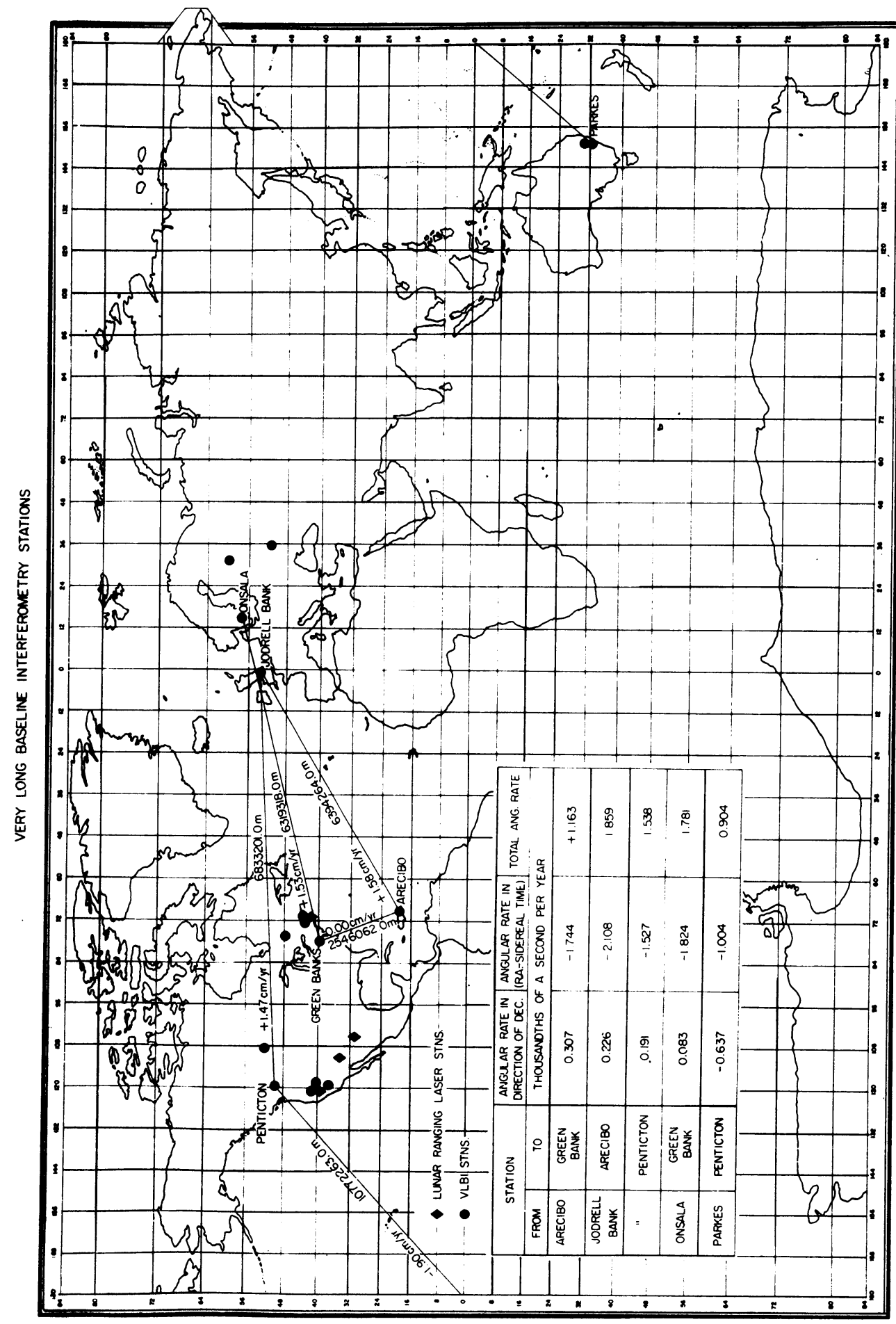

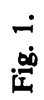




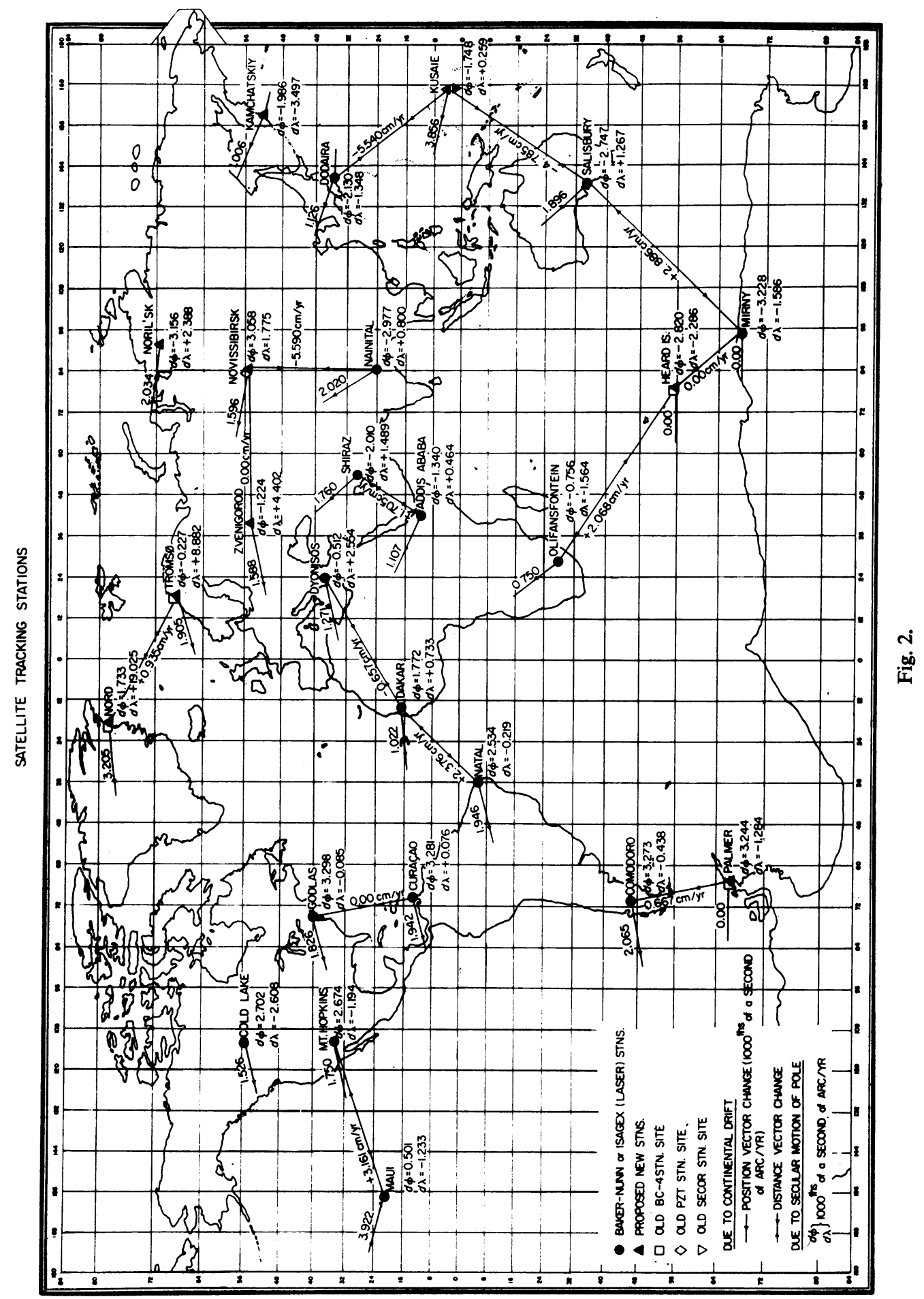




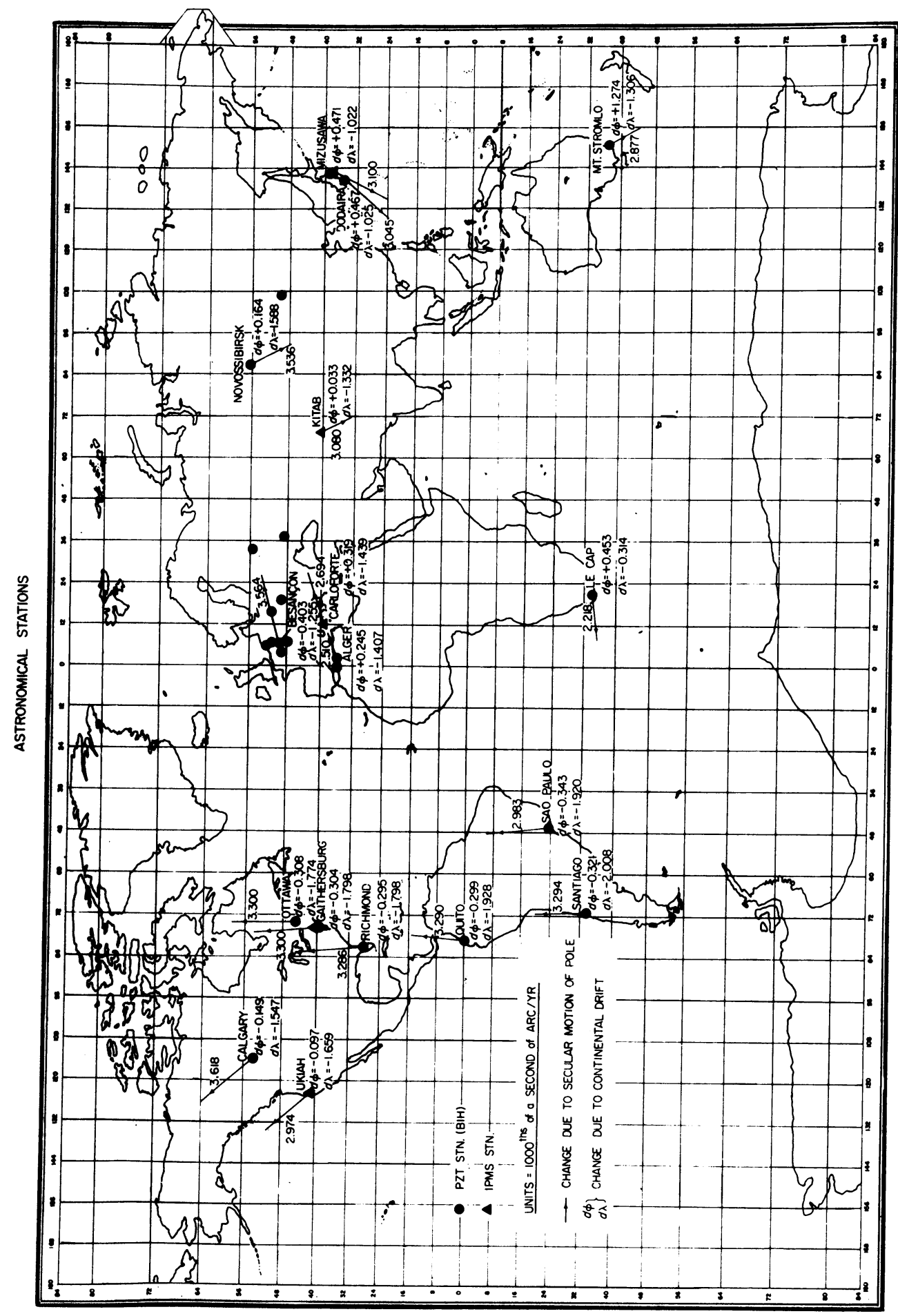

$\stackrel{\infty}{\infty}$ 
the largest predicted rate of change of length. However, a measured change in the length of this baseline would not be a completely satisfactory confirmation of continental drift, since the two stations are not on contiguous tectonic blocks.

Figure 2 depicts the predicted annual rates of change of position due to continental drift and due to secular motion of the pole at selected satellite tracking stations. Most of these stations are participating in the ISAGEX experiment with either Baker-Nunn cameras or laser ranging equipment. However, several proposed stations have been added to provide at least three well separated stations on each of the six tectonic blocks. The proposed stations at Nord, Tromsø, Palmer, and Heard Island, are placed at sites previously occupied by BC-4 cameras. Zvenigorod, and Novosibirsk are placed at sites of astronomical observations. Kusai is the site of a former SECOR station. Noril'sk and Kamchatsky are new stations. The values $(\mathrm{d} \varphi, \mathrm{d} \lambda)$ written next to the station designations indicate expected changes in the coordinates due to the presently believed rate of secular motion of the pole.

Figure 3 depicts the predicted rates of change of position at selected astronomical observatories. The vectors indicate the expected changes due to the secular polar motion, while the numbers $(\mathrm{d} \varphi, \mathrm{d} \lambda)$ written next to the station designations are expected coordinate changes due to continental drift.

\section{Required Observations}

In order to judge how large a time interval must elapse before changes in position would be detectable, it is necessary to assume certain precisions for each of the observing schedules. The precision with which the VLBI baselines (or the coordinates of lunar ranging stations) will be measured in the next few years is assumed to be $15 \mathrm{~cm}$ (Kaula, 1969, p. 7-6). Within a decade, it should be possible to measure the length of the baselines to $2 \mathrm{~cm}$, and to determine their orientation relative to celestial radio sources to $0.001 \mathrm{sec}$ of arc (Kaula, 1969, p. 7-9, p. 2-1).

The precision of presently operating laser ranging instruments appears to be about one meter. However, ranging precision as high as $15 \mathrm{~cm}$ should be achieved within a few years, and a precision of $2 \mathrm{~cm}$ should be available within a decade (Kaula, 1969, p. 2-9, p. 7-6, p. 7-9). The precision of optical observations to satellite is of the order of $1^{\prime \prime}$.

The precision assumed for observations of latitude and longitude from the astronomical observatories is $0.015 \mathrm{sec}$ of arc from one night of observations (Mueller, 1969 , p. 401). No improvement in these accuracies is foreseen.

If the motions of the continents and of the pole are really continuous phenomena, then significant changes in the relative and absolute positions of the observing stations should be detectable if the positions are reobserved after a sufficiently long time interval. The time bases in Tables III and IV are computed for the length of time it would take for the predicted motion to equal the uncertainty with which the motion can be observed ( $1 \sigma$ level), and for the length of time required before the existence of motion can be confirmed with a high degree of statistical certainty ( $3 \sigma$ level). 
TABLE III

Time base for reobservations of VLBI baselines

\begin{tabular}{|c|c|c|c|}
\hline From & To & $1 \sigma$ level & $3 \sigma$ level \\
\hline & \multicolumn{3}{|c|}{$\sigma\{$ length $\}=15 \mathrm{~cm}$ (immediate) } \\
\hline Arecibo & Green Bank & $10 \mathrm{yr}$ & $30 \mathrm{yr}$ \\
\hline Jodrell Bank & Arecibo & $10 \mathrm{yr}$ & $30 \mathrm{yr}$ \\
\hline Jodrell Bank & Penticton & $10 \mathrm{yr}$ & $30 \mathrm{yr}$ \\
\hline Onsala & Green Bank & $10 \mathrm{yr}$ & $30 \mathrm{yr}$ \\
\hline \multirow[t]{2}{*}{ Parkes } & Penticton & $8 \mathrm{yr}$ & $24 \mathrm{yr}$ \\
\hline & \multicolumn{3}{|c|}{$\sigma\{$ length $\}=2 \mathrm{~cm}$ (eventual) } \\
\hline Arecibo & Green Bank & $1.3 \mathrm{yr}$ & $4 \mathrm{yr}$ \\
\hline Jodrell Bank & Arecibo & $1.3 \mathrm{yr}$ & $4 \mathrm{yr}$ \\
\hline Jodrell Bank & Penticton & $1.3 \mathrm{yr}$ & $4 \mathrm{yr}$ \\
\hline Onsala & Green Bank & $1.3 \mathrm{yr}$ & $4 \mathrm{yr}$ \\
\hline \multirow[t]{2}{*}{ Parkes } & Penticton & $1.1 \mathrm{yr}$ & $3 \mathrm{yr}$ \\
\hline & \multicolumn{3}{|c|}{$\sigma\{$ orientation $\}=0.001$ (eventual) } \\
\hline Arecibo & Green Bank & $0.8 \mathrm{yr}$ & $2.6 \mathrm{yr}$ \\
\hline Jodrell Bank & Arecibo & $0.5 \mathrm{yr}$ & $1.6 \mathrm{yr}$ \\
\hline Jodrell Bank & Penticton & $0.7 \mathrm{yr}$ & $2.0 \mathrm{yr}$ \\
\hline Onsala & Green Bank & $0.6 \mathrm{yr}$ & $1.7 \mathrm{yr}$ \\
\hline Parkes & Penticton & $1.1 \mathrm{yr}$ & $3.3 \mathrm{yr}$ \\
\hline
\end{tabular}

TABLE IV

Time bases for reobservation of relative positions between satellite observing stations

\begin{tabular}{llcc}
\hline From & To & $1 \sigma$ level & $3 \sigma$ level \\
\hline & $\sigma$ \{distance & $15 \mathrm{~cm}$ (immediate) & \\
Maui & Mt. Hopkins & $5 \mathrm{yr}$ & $14 \mathrm{yr}$ \\
Dakar & Natal & $6 \mathrm{yr}$ & $19 \mathrm{yr}$ \\
Dakar & Dyonisos & $23 \mathrm{yr}$ & $68 \mathrm{yr}$ \\
Addis Ababa & Shiraz & $9 \mathrm{yr}$ & $26 \mathrm{yr}$ \\
Mirny & Salisbury & $5 \mathrm{yr}$ & $16 \mathrm{yr}$ \\
Nord & Tromsø & $16 \mathrm{yr}$ & $48 \mathrm{yr}$ \\
Kusai & Salisbury & $3 \mathrm{yr}$ & $9 \mathrm{yr}$ \\
Kusai & Dodaira & $3 \mathrm{yr}$ & $8 \mathrm{yr}$ \\
Nainital & Novosibirsk & $3 \mathrm{yr}$ & $8 \mathrm{yr}$ \\
\hline
\end{tabular}

\section{Conclusions}

Present astronomical and satellite observing stations are not in locations that would be the most advantageous for the detection of relative motions between the continental blocks. Of the lines that are being observed with lasers at both ends as part of the ISAGEX experiment, a rate of change of relative position of more than $2 \mathrm{~cm} / \mathrm{yr}$ is predicted only for the line between Dakar and Natal. There is also a line with lasers at both ends between Dodaira and Guam. However, Guam is on the Asian side of the Mariana Trench, which the Le Pichon model considers to be the boundary between the Eurasian and Pacific blocks, and so the model predicts no change in the 
distance between Dodaira and Guam. If this station were moved to the former SECOR site at Kusai, it would be possible to observe a line between Dodaira and Kusai, where the predicted rate of change is $-5.5 \mathrm{~cm} / \mathrm{yr}$. The lines between several other pairs of stations, such as Nainatal - Novosibirsk and Salisbury - Kusai, are also expected to change by about $5 \mathrm{~cm} / \mathrm{yr}$. If the lengths of these lines are observed to $15 \mathrm{~cm}$ within a few years, it should be possible to obtain a strong confirmation of relative motion between continents with a time base of less than a decade.

As far as the astronomical observations are concerned, it may be noted from Figure 3 that the expected continental drift will change the coordinates of most observatories mostly in longitude, therefore longitude observations on a continuous basis are of the utmost importance.

Although detection of relative motion between stations on different tectonic blocks is of greatest interest, detection of a lack of relative motion between stations on the same block is of equal importance. The Le Pichon model of global tectonic requires that the blocks be rigid, subject to deformation only at their boundaries. Thus, it is important to determine the extent to which the continents do move as rigid blocks if detection of motion between blocks is to confirm the existence of continental drift. Confirmation of the rigidity of the blocks will require measurements of the relative positions of several additional well placed stations on each block.

\section{References}

Arur, M. G. and Mueller, Ivan I.: 1971, J. Geophys. Res. 76, No. 8.

Centre National d'Etudes Spatial: 1971, 'International Satellite Geodesy Experiment (ISAGEX) Experiment Plan'.

Kaula, William M. (ed.): 1969, 'Solid Earth and Ocean Physics', Report of a Study at Williamstown, Massachusetts, sponsored by NASA-Electronics Research Center and MIT Measurement Systems Laboratory.

Le Pichon, X.: 1968, J. Geophys. Res. 73, No. 12.

Le Pichon, X.: 1970, J. Geophys. Res. 75, No. 14.

Mueller, I. I.: 1969, Spherical and Practical Astronomy as Applied to Geodesy, Frederick Ungar Publ. Comp., New York.

\section{DISCUSSION}

D. G. Currie: Although it does not have the advantage of your careful analysis, I would suggest that the possible stations for laser ranging to the Moon should be included in any Symposium Resolution. This is because the current accuracy of the laser ranging to the Moon has now an accuracy of $15 \mathrm{~cm}$ and should have an accuracy of $3 \mathrm{~cm}$ in $3 \mathrm{yr}$, and a resolution of the type you suggested would encourage the establishment of such stations in the interested countries.

I. I. Mueller: The two stations in the United States have been included, the stations in France and Japan were not included because of a lack of knowledge of their operational status.

H. Takeuchi: If we do not know that the continents are drifting and we analyse the data, there will appear an apparent polar wandering. What will be the direction and magnitude of this apparent wandering averaged over all ILS and BIH stations?

I. I. Mueller: Not yet made the calculation.

P. Melchior: I don't think that the Earth's crust can be considered as made of only six rigid blocks. Earth Tide measurements show many more regional anomalies for example in the phase lag of vertical component. 